\title{
BMJ Open Research priorities for the management of complex fractures: a UK priority setting partnership with the James Lind Alliance
}

Christopher Patrick Bretherton (10) , ${ }^{1}$ Henry A Claireaux, ${ }^{1}$ Jonathan Gower, ${ }^{2}$ Shan Martin, ${ }^{3}$ Angela Thornhill, ${ }^{4}$ Louise Johnson, ${ }^{5}$ Lucy Silvester, ${ }^{6}$ Rebecca Samantha Kearney (i) , ${ }^{7}$ Mark Baxter, ${ }^{8}$ Paul Dixon, ${ }^{9}$ Victoria Giblin, ${ }^{10}$ Xavier Luke Griffin (D) , ${ }^{11}$ William Eardley ${ }^{9}$

\section{ABSTRACT}

Objective To determine research priorities for the management of complex fractures, which represent the shared priorities of patients, their families, carers and healthcare professionals.

Design/setting A national (UK) research priority setting partnership.

Participants People who have experienced a complex fracture, their carers and relatives, and relevant healthcare professionals and clinical academics involved in treating patients with complex fractures. The scope includes open fractures, fractures to joints broken into multiple pieces, multiple concomitant fractures and fractures involving the pelvis and acetabulum.

Methods A multiphase priority setting exercise was conducted in partnership with the James Lind Alliance over 21 months (October 2019 to June 2021). A national survey asked respondents to submit their research uncertainties which were then combined into several indicative questions. The existing evidence was searched to ensure that the questions had not already been sufficiently answered. A second national survey asked respondents to prioritise the research questions. A final shortlist of 18 questions was taken to a stakeholder workshop, where a consensus was reached on the top 10 priorities.

Results A total of 532 uncertainties, submitted by 158 respondents (including 33 patients/carers) were received during the initial survey. These were refined into 58 unique indicative questions, of which all 58 were judged to be true uncertainties after review of the existing evidence. 136 people (including 56 patients/carers) responded to the interim prioritisation survey and 18 questions were taken to a final consensus workshop between patients, carers and healthcare professionals. At the final workshop, a consensus was reached for the ranking of the top 10 questions.

Conclusions The top 10 research priorities for complex fracture include questions regarding rehabilitation, complications, psychological support and return to liferoles. These shared priorities will now be used to guide funders and teams wishing to research complex fractures over the coming decade.
Strengths and limitations of this study

- Use of established and transparent James Lind Alliance methodology.

- Survey responses were received from across the UK and from a range of patients and healthcare providers.

- The SARS-CoV-2 pandemic limited the use of volunteers to gather in-person responses from patients.

\section{BACKGROUND}

Complex fractures are injuries that involve severe breaks to a bone or multiple bones. They can involve skin loss and compound injuries of nerves, blood vessels and other tissues. They often require specialist treatment and can be associated with prolonged rehabilitation and disability. ${ }^{1-3}$ The National Institute for Health and Care Excellence (NICE) guidance on complex fractures encompasses fractures of the pelvis, fractures to joints broken into multiple pieces, and open fractures in which skin loss or significant tissue damage occurs. ${ }^{4}$

Complex fractures make up the minority of the two million fractures treated in England each year but are associated with significant morbidity and are a large burden on healthcare resources. ${ }^{5}$ They often involve highenergy transfer mechanisms, such as road traffic accidents. ${ }^{6}$ Multiple injuries or fractures can be sustained at the same time. In elderly patients, the same spectrum of severe injuries can occur with lower-energy transfer accidents such as trips and falls from standing height. ${ }^{78}$ The treatment of complex fractures is often complicated and usually involves multiple healthcare professionals and specialists. $^{14}$ 
High-quality research into complex fractures is lacking, this is partly because they are less common and often have concomitant injuries and/or comorbidities. The introduction of the UK Major Trauma Network, utilisation of the Trauma Audit and Research Network and the development of novel methodical approaches have seen a rise in the number of trials in urgent or emergency care conducted in the National Health Service over the last 5 years. ${ }^{910}$ The UK now has the infrastructure to deliver high-quality research in the emergency setting, and so there is a pressing need to determine the research priorities for patients with complex fractures and their families. ${ }^{911}$ This Priority Setting Partnership (PSP) is the first to investigate these priorities systematically.

The James Lind Alliance (JLA) is an independent, non-profit organisation hosted by the National Institute for Health Research (NIHR). The JLA is committed to the principles of inclusivity, transparency and equal involvement of patients, carers and health professionals in research prioritisation. ${ }^{12}$ The aim of this work was to establish the research priorities for adults with complex fractures which represent the shared interests and priorities of patients, their families and friends, carers and healthcare professionals.

\section{METHODS}

The Complex Fractures PSP was conducted in accordance with the JLA process ${ }^{12}$ and was undertaken over 21 months (October 2019 to June 2021) (see figure 1).

\section{Steering group and partner organisations}

Steering group members were recruited from professional and charitable organisations, including patients, doctors, clinical academics and allied healthcare professionals from around the UK. A JLA advisor (JG) guided the process, acting as a neutral facilitator to promote equal contributions from patients and healthcare professionals and to ensure JLA principles and methodology were followed. The information specialist (CPB) designed the

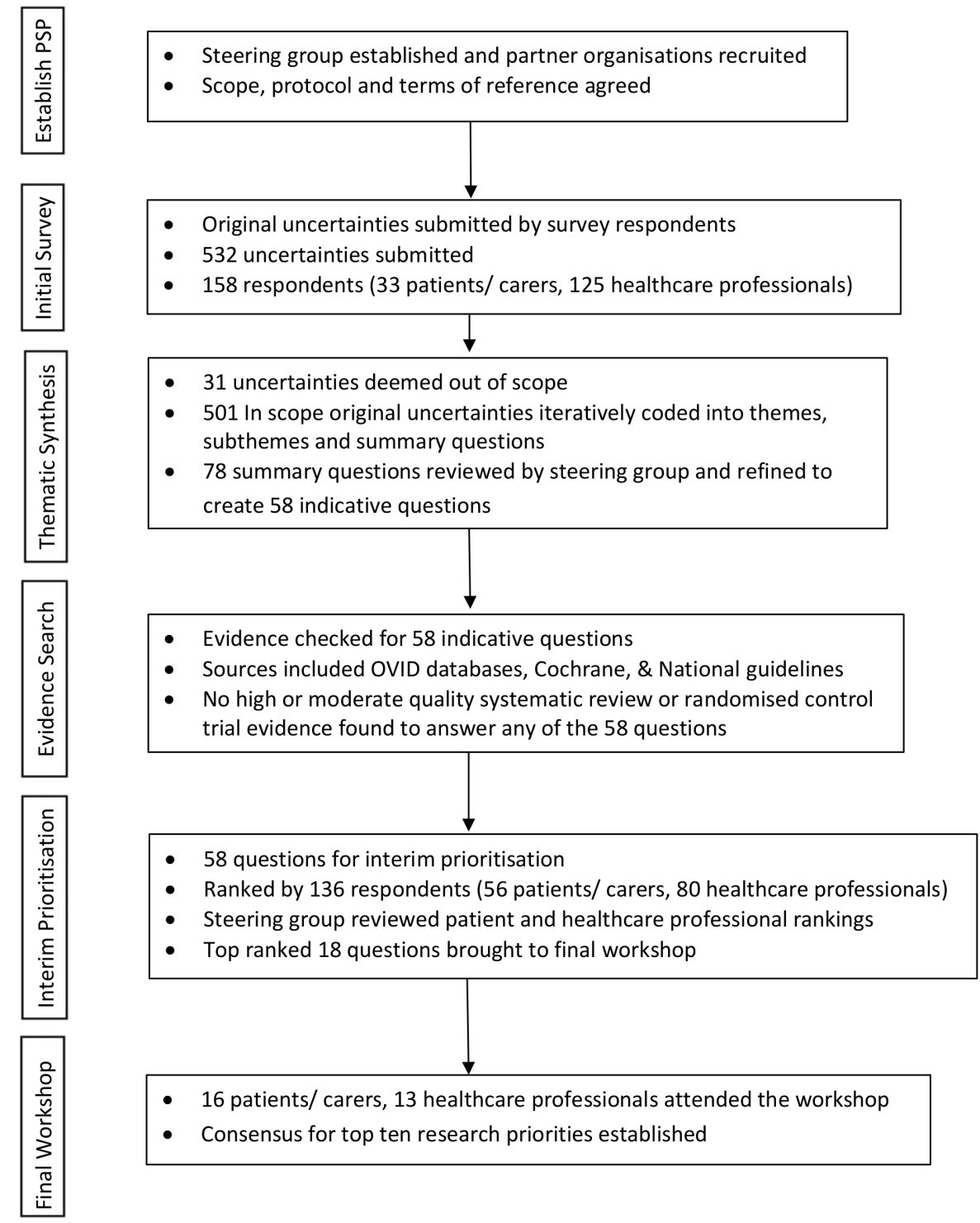

Figure 1 Flow chart of priority setting partnership process. PSP, Priority Setting Partnership 
surveys, managed the data and performed the analysis. Each step was overseen by the steering group.

\section{Scope}

The scope of the PSP mirrored the conditions included in the NICE guidance for Complex Fractures. ${ }^{4}$ This included but was not limited to open fractures, fractures to joints broken into multiple pieces, multiple concomitant fractures and fractures involving the pelvis and acetabulum. Fractures associated with neurological injury, vascular injury or compartment syndrome were also included. Fractures included in the NICE guidelines for non-complex fractures were excluded. ${ }^{13}$ Fractures in children, emergency department and prehospital care and isolated hand injuries were also excluded as these fall within existing or planned PSPs. Decisions about whether submissions were in or out-of-scope were made by the information specialist and verified by the steering group.

\section{Initial survey and identification of themes}

The steering group designed an initial survey, asking respondents to submit their free-text research uncertainties for complex fractures, prompting them to consider early treatment and aspects of recovery up until 12 months from injury. Demographic information was also collected. The survey was available in paper and online formats (see online supplemental file 1A 'Initial Survey'). The survey was launched at the multi-disciplinary UK Trauma Trials Day and Orthopaedic Trauma Society conference on 15 January 2020. The survey was disseminated through partner organisations, social media and to patients in hospital wards and clinics (see online supplemental file 2 'Partner organisations'). Face-to-face patient participation was initially challenging due to the SARS-CoV-2 pandemic and so the initial survey remained open till August 2020.

All submissions were analysed by the information specialist, first splitting longer submissions into discrete components based on topic transitions. After a period of data immersion, responses were coded into themes, subthemes and then into summary questions. Each initial submission and its corresponding theme and summary question were verified by at least two members of the steering group, including a patient representative. This verification process involved reading each initial submission and corresponding summary question to ensure the summary question reflected the initial submission, any disagreements were discussed at a steering group meeting to reach consensus.

\section{Creation of indicative questions and evidence checking}

The steering group met to review all the themes and summary questions in turn. Similar questions were amalgamated into 'indicative questions', ensuring each of the original submissions were represented. Each indicative question was reviewed during the steering group meeting for readability and to ensure the language was understandable to patients and stakeholder groups.
A literature review was undertaken to ensure each indicative question was a 'true' uncertainty and had not already been sufficiently answered by research. HAC searched PubMed, Cumulative Index Nursing Allied Health, British Nursing Index, Embase, Medline, PsycINFO, Google Scholar, the WHO International Clinical Trials Registry Platform Search Portal, the US National Institute of Health Trials Registry, ISRCTN Registry and Published UK national guidelines ${ }^{4}$ (see search strategy in online supplemental file 3 'Question verification form').

The indicative questions were considered to be "unanswered' if no recent (within the past 5 years) systematic reviews of research evidence or randomised controlled trials demonstrating high-quality or moderate-quality evidence for the question existed. ${ }^{14}$ The steering group reviewed each indicative question and the available summarised evidence to verify it was a true uncertainty. Where there were ongoing randomised controlled trials, two academic surgeons (WE and XLG) reviewed the studies to determine if they were likely to provide definitive evidence for the indicative question.

\section{Interim prioritisation}

A second survey asked respondents to pick their top 10 priorities from the indicative questions. The survey was distributed in online and paper formats through the same channels as the initial survey between 12 January 2021 and 1 April 2021 (see online supplemental file 1B) 'Interim Survey'). Separate rankings for patients (and their relatives and carers) and healthcare professionals were generated to account for a disparate proportion of responses and promote equal weighting between the stakeholder groups. The geometric means were calculated and combined to give the interim rankings. The steering group reviewed the rankings and chose a manageable list of questions to discuss at the final workshop.

\section{Final consensus workshop}

On 8 June 2021, a virtual 1-day workshop brought together patients, carers and healthcare professionals to determine the 'Top 10' research priorities for complex fractures. A sampling framework was used to invite and finally select participants from earlier stages of the PSP and additional volunteers from patient organisations. Within the sampling framework age, gender, geography, ethnicity and professional and personal experience were taken into consideration.

Prior to the workshop, participants were sent introductory materials and videos and asked to rank the questions from highest to lowest priority. During the workshop, participants were split into four groups of $7-8$ comprising an equal distribution of patient representatives and healthcare professionals. Each group was facilitated by a JLA advisor who asked participants to list their highest and lowest priorities and discuss their rationale. An iterative ranking process continued with participants allocated to new, equally balanced small groups to exchange views, with all participants encouraged to contribute. During the 
breaks, JLA advisors combined the rankings for each group to generate an updated list for subsequent discussion. After the final round, the JLA advisors presented the final combined rankings, and the participants reflected on the final consensus priorities.

\section{Patient and public involvement}

Patient and carer representatives were engaged throughout the process. They helped define the scope and were involved in the review of all patient-facing media. They were involved in all steering group meetings and decisions. They collaborated with patient organisations and helped to reach a diverse range of patient and carers groups for the surveys and final workshop. Two patient steering group members attended the final workshop to help link the indicative questions to the underlying submissions from which they were derived. Patient representatives will help disseminate the PSP findings and work with patient and charitable organisations to develop discrete research questions from the final priorities to take forward for funding.

\section{RESULTS}

\section{Initial survey and evidence checking}

A total of 158 responses were received from 26 Patients, 7 relatives/carers, 119 healthcare professions and 6 preferred not to say. The median age of respondents was 45 (range 24-73), 91 (57.6\%) were male, 55 (34.8\%) were female, $12(7.6 \%)$ preferred not to say. A total of 113 identified as white $(71.5 \%), 26(16.5 \%)$ as minority ethnic, $19(12.0 \%)$ preferred not to say. Respondents were from England (118), Wales (2), Northern Ireland (3) and Scotland (3), with 31 unknown. They submitted a total of 532 unique research uncertainties. After removal of out-of-scope submissions 501 remained. Out-of-scope submissions can be viewed in online supplemental file 4 'Out-of-scope initial submissions'.

A total of 78 summary questions were reviewed by the steering group and consolidated to create 58 indicative questions. No questions were found to be sufficiently answered by existing research during evidence checking and all progressed to interim prioritisation.

\section{Interim survey}

A total of 136 responses were received from 80 (58.8\%) healthcare professionals, $53(39.0 \%)$ patients and 3 $(2.2 . \%)$ relatives/carers. $72(52.9 \%)$ female, $62(45.6 \%)$ male, $2(1.5 \%)$ preferred to self-describe. $116(85.3 \%)$ identified as white, $17(12.5 \%)$ as minority ethnic, 3 $(2.2 \%)$ preferred not to say. There were responses from across the UK including England (94), Wales (7), Northern Ireland (4) and Scotland (2), with 29 unknown.

The steering group reviewed the rankings and based on previous experience of PSP workshops it was agreed that 18 questions would be taken forwards to the final workshop. There was similarity between the top ranked questions for healthcare professionals and patients,

\section{Box 1 Top 10 UK research priorities for complex fractures}

1. What is the best way to reduce the risk of infection after complex fractures?

2. What is the optimal outpatient rehabilitation strategy for patients with complex fractures?

3. What psychological support would be useful for patients with complex fractures and when?

4. Is it possible to determine which patients will develop complications, arthritis and poor functional outcomes after complex fractures?

5. What are the options for preventing and treating chronic (longterm) pain after complex fractures?

6. What is important to patients recovering from complex fractures?

7. What additional care and support is helpful for patients being discharged from hospital after a complex fracture?

8. When is it better to replace, fix or fuse fractures around the ankle, knee or acetabulum (hip socket)?

9. Can peer support (from other patients) be used to help patients with complex fractures?

10. Can patients be provided with expected recovery times for functional recovery and return to life roles after complex fractures?

with 9 of the top 10 ranked questions for both groups featuring in the 18 questions taken to the final prioritisation workshop.

\section{Final consensus workshop}

The final workshop was attended by 13 healthcare professionals (including surgeons $(\mathrm{n}=5)$, psychologists $(n=2)$, physiotherapists $(n=2)$, orthogeriatricians $(n=2)$, an anaesthetist and an occupational therapist) and 16 patient representatives (14 had personal experience of complex fractures and 2 were relatives/carers). This included 4 healthcare professionals and 2 patient representatives from the steering group.

The order of the final 10 priorities was agreed by consensus. They are shown in box 1: final top 10 research priorities. The full list of the top 18 can be viewed in online supplemental file 5: 'Indicative questions 1-18 and evidence summary'. The indicative questions that fell outside of the 18 discussed at the priority setting workshop can be viewed in online supplemental file 6: 'Indicative Questions 19-58'.

\section{DISCUSSION}

We have reported the results of a UK Priority Setting Partnership to establish the top ten priorities for research in complex fractures. This JLA process has helped ensure the top ten reflect the shared priorities of patients, their carers and relatives, and healthcare professionals. The questions reflect a shift in priorities seen in other musculoskeletal PSP's, ${ }^{15} 16$ in which the traditional researcher-led questions comparing surgical techniques have been largely replaced by holistic, patient-centric questions. Greater attention to psychological support, informing expectations for recovery and ensuring research outcomes are important to patients are commonly featured priorities. 
The prevention and treatment of infection, minimising its significant morbidity, is another common shared priority.

The strengths of this study are that it followed the robust JLA methodology with independent facilitation by a JLA Adviser, maintaining the principles of transparency and equal inclusion. ${ }^{12}$ This is the first study that has sought to understand what makes a fracture 'complex' and reports national research priorities for their treatment and ongoing care. It builds on the NICE description of 'complex fractures', refining the definition to be more than a set of individual fractures. Rather, the definition used in this study identifies patients that are likely to require numerous treatments, over prolonged periods, with input from multiple healthcare teams and services. ${ }^{4}$ The respondents, the steering group and the approach taken by this priority setting partnership reflect the multidisciplinary approach required to care for patients with these challenging injuries. The recent advent of the UK Major Trauma network allowed wide dissemination of the surveys and the resulting broad geographical spread of responses ensures that the priorities are representative of a national viewpoint. ${ }^{9}$

This study has limitations. First, previous PSPs have used patient volunteers to disseminate the survey and gather research uncertainties, ${ }^{15}$ the use of these in-person methods was not possible due to the SARSCoV-2 pandemic. Other challenges included engaging patients during outpatient appointments: Most fracture-related and musculoskeletal conditions have high volume follow-up clinics, however, complex fracture clinics typically have fewer, longer appointments, in which patients have multiple outcome surveys to complete, and so survey burden becomes an issue. ${ }^{9}$ The number of patient responses is, therefore, lower than other musculoskeletal PSPs. ${ }^{15} 16$ This partly reflects the lower incidence of complex fractures compared with 'non-complex' fractures. To limit bias from the disproportionate representation of healthcare professionals, we used separate rankings and combining of geometric means for the interim rankings. We also ensured a balanced composition of participants at the final workshop.

Discussions at the final workshop recognised that there may be some challenges with addressing these research priorities and that going forwards early international collaboration may be advisable and future PSP's may wish to consider this at the priority setting stage. Lessons learnt from conducting the Complex Fractures PSP during a pandemic may be transferable to future, international PSP's. We found arranging for steering group members and workshop participants to share their biographies in advance saved time during virtual meetings and enhanced interaction. Having a wide geographic range of enthusiastic collaborators to approach patients in their local hospital appeared more effective than engagement through online methods. Finally, having a General Data Projection Regulation compliant method for collecting respondent details to enable recontact for later phases was crucial, especially when gathering patient responses proved challenging.

This PSP has highlighted new research priorities for complex fractures. Investigating these questions will require a range of research methodologies, beyond conventional Randomised Controlled Trials comparing implant A versus implant B. The steering group will disseminate these findings widely and work with research funding bodies and charitable organisations to develop research questions in partnership with patient representatives who contributed to identifying the priorities. The results of this priority setting partnership can be used to guide research funding bodies and the wider research community in advancing the quality of care for patients with complex fractures.

\section{Author affiliations}

${ }^{1}$ Nuffield Department of Orthopaedics, Rheumatology and Musculoskeletal Sciences, University of Oxford, Oxford, UK

'James Lind Alliance, Southampton, UK

${ }^{3}$ Patient Representative, Wessex, UK

${ }^{4}$ Patient Representative, East Midlands, UK

${ }^{5}$ Clinical Psychology, Leeds General Infirmary, Leeds, UK

${ }^{6}$ Physiotherapy, University Hospitals Coventry and Warwickshire NHS Trust, Coventry, UK

${ }^{7}$ Warwick Clinical Trials Unit, University of Warwick, Coventry, UK

${ }^{8}$ Orthogeriatrics, University Hospital Southampton NHS Foundation Trust, Southampton, UK

${ }^{9}$ Trauma \& Orthopaedic Surgery, James Cook University Hospital, Middlesbrough, UK

${ }^{10}$ Plastic Surgery, Sheffield Teaching Hospitals NHS Foundation Trust, Sheffield, UK

${ }^{11}$ Trauma \& Orthopaedic Surgery, Queen Mary University of London, London, UK

Twitter Christopher Patrick Bretherton @BrethertonC and Xavier Luke Griffin @ xlgriffin

Acknowledgements The steering group would like to thank to all the patients, carers, families, friends, healthcare professionals and partner organisations who contributed to this work. Thank you to Lisa Robertson for coordinating the initial stages of the PSP. The steering group gives thanks to all other participants/ facilitators at the final workshop for their hard work to achieve consensus on the final top ten (Amber Young, Caroline James, Claudia Myler, David Wallace, Debbie MacPhee, Douglas Bairstow, Farzana Kausir, Helga Ruff, Joe Alsousou, John Davis, Josephine Madden, Julie Craig, Maria King, Moira Jordan, Nicholas Crosby, Peter Campaigne, Russel Neighbour, Scott Johnson, Victoria Lyle, Wareth Maamoun, Katherine Cowan, Maryrose Tarpey, Susannah Kinsella). Special thanks to Day One Trauma Support Charity who helped disseminate the surveys and recruited four patient representatives for the final workshop.

Contributors All authors (CPB, HAC, JG, SM, AT, LJ, LS, RSK, MB, PD, VG, XLG and WE) made significant contributions to the design, implementation and delivery of this research. HAC conducted the evidence searching. CPB analysed the data and prepared the initial manuscript. All authors have read and approved the final version of this manuscript. CPB is responsible for the overall content as the guarantor.

Funding This work was supported by AOUK\&l, grant number HFR03160.

Competing interests RSK is a member of the UK NIHR HTA CET board, NIHR ICA Doctoral panel, chair of the NIHR RfPB board and holder of a NIHR Senior Fellowship award. RSK has been awarded current and previous NIHR and vs Arthritis research grants. RSK is co-investigator on an NIHR funded study receiving additional support from Stryker Ltd.XLG is funded by an NIHR Clinician Scientist Award.

Patient consent for publication Not required.

Ethics approval This work did not require ethics approval as per the guidance published by the NHS National Patient Safety Agency National Research Ethics Service. Participant informed consent for survey respondents and workshop attendees was not required or obtained.

Provenance and peer review Not commissioned; externally peer reviewed. 
Data availability statement All data relevant to the study are included in the article or uploaded as online supplemental information. Online supplemental data including all indicative questions and out of scope submissions can be found on the JLA website at: https://www.jla.nihr.ac.uk/priority-setting-partnerships/complexfractures/

Supplemental material This content has been supplied by the author(s). It has not been vetted by BMJ Publishing Group Limited (BMJ) and may not have been peer-reviewed. Any opinions or recommendations discussed are solely those of the author(s) and are not endorsed by BMJ. BMJ disclaims all liability and responsibility arising from any reliance placed on the content. Where the content includes any translated material, BMJ does not warrant the accuracy and reliability of the translations (including but not limited to local regulations, clinical guidelines, terminology, drug names and drug dosages), and is not responsible for any error and/or omissions arising from translation and adaptation or otherwise.

Open access This is an open access article distributed in accordance with the Creative Commons Attribution Non Commercial (CC BY-NC 4.0) license, which permits others to distribute, remix, adapt, build upon this work non-commercially, and license their derivative works on different terms, provided the original work is properly cited, appropriate credit is given, any changes made indicated, and the use is non-commercial. See: http://creativecommons.org/licenses/by-nc/4.0/.

\section{ORCID iDs}

Christopher Patrick Bretherton http://orcid.org/0000-0001-9569-0734

Rebecca Samantha Kearney http://orcid.org/0000-0002-8010-164X

Xavier Luke Griffin http://orcid.org/0000-0003-2976-7523

\section{REFERENCES}

1 Coccolini F, Kluger Y, Moore EE, et al. Trauma quality indicators: internationally Approved core factors for trauma management quality evaluation. World J Emerg Surg 2021;16:6.

2 Keene DJ, Knight R, Bruce J, et al. Chronic pain with neuropathic characteristics after surgery for major trauma to the lower limb: prevalence, predictors, and association with pain severity, disability, and quality of life in the UK WHiST trial. Bone Joint $J$ 2021;103B:1047-54.
3 Rees S, Tutton E, Achten J, et al. Patient experience of long-term recovery after open fracture of the lower limb: a qualitative study using interviews in a community setting. BMJ Open 2019;9:e031261.

4 NICE. Overview / fractures (complex). Assessment and management | Guidance | NICE 2021 https://www.nice.org.uk/guidance/ng37

5 Donaldson LJ, Reckless IP, Scholes S, et al. The epidemiology of fractures in England. J Epidemiol Community Health 2008;62:174-80.

6 Roberts Z, Collins J-A, James D, et al. Epidemiology of adolescent trauma in England: a review of TARN data 2008-2017. Emerg Med J 2020;37:25-30.

7 Lesko K, Deasy C. Low falls causing major injury: a retrospective study. Ir J Med Sci 2020;189:1435-43.

8 Dixon JR, Lecky F, Bouamra O, et al. Age and the distribution of major injury across a national trauma system. Age Ageing 2020;49:218-26.

9 Moran CG, Lecky F, Bouamra O, et al. Changing the System - Major Trauma Patients and Their Outcomes in the NHS (England) 2008-17. EClinicalMedicine 2018;2-3:13-21.

10 Costa ML, Achten J, Bruce J, et al. Effect of negative pressure wound therapy vs standard wound management on 12-month disability among adults with severe open fracture of the lower limb: the WOLLF randomized clinical trial. JAMA 2018;319:2280-8.

11 National Institute for Health, 2015. Going the extra mile improving the nation's health and wellbeing through public involvement in research 2021;5 www. nihr. ac. uk/ about- us/ documents/ Extra\% 20 Mile2. pdf

12 The James Lind Alliance. James Lind alliance, 2021. Available: https://www.jla.nihr.ac.uk/

13 National Institute of Health and Care Excellence. Fractures (noncomplex) assessment and management 2016.

14 Higgins JPT, Altman DG, Gøtzsche PC, et al. The Cochrane collaboration's tool for assessing risk of bias in randomised trials. BMJ 2011;343:d5928.

15 Fernandez MA, Arnel L, Gould J, et al. Research priorities in fragility fractures of the lower limb and pelvis: a UK priority setting partnership with the James Lind alliance. BMJ Open 2018;8:e023301.

16 Sheehan WJ, Williams MA, Paskins Z, et al. Research priorities for the management of broken bones of the upper limb in people over 50: a UK priority setting partnership with the James Lind alliance. BMJ Open 2019;9:e030028. 